ARTICLE

Received 25 Dec 2016 | Accepted 6 Apr 2017 | Published 30 May 2017

\title{
Coherent control of the silicon-vacancy spin in diamond
}

Benjamin Pingault ${ }^{1}$, David-Dominik Jarausch ${ }^{1}$, Christian Hepp ${ }^{1}$, Lina Klintberg${ }^{1}$, Jonas N. Becker ${ }^{2}$, Matthew Markham ${ }^{3}$, Christoph Becher ${ }^{2} \&$ Mete Atatüre $^{1}$

Spin impurities in diamond have emerged as a promising building block in a wide range of solid-state-based quantum technologies. The negatively charged silicon-vacancy centre combines the advantages of its high-quality photonic properties with a ground-state electronic spin, which can be read out optically. However, for this spin to be operational as a quantum bit, full quantum control is essential. Here we report the measurement of optically detected magnetic resonance and the demonstration of coherent control of a single silicon-vacancy centre spin with a microwave field. Using Ramsey interferometry, we directly measure a spin coherence time, $T_{2}{ }^{\star}$, of $115 \pm 9 \mathrm{~ns}$ at $3.6 \mathrm{~K}$. The temperature dependence of coherence times indicates that dephasing and decay of the spin arise from single-phonon-mediated excitation between orbital branches of the ground state. Our results enable the silicon-vacancy centre spin to become a controllable resource to establish spin-photon quantum interfaces.

\footnotetext{
${ }^{1}$ Cavendish Laboratory, University of Cambridge, JJ Thomson Avenue, Cambridge CB3 OHE, UK. ${ }^{2}$ Fachrichtung 7.2 (Experimentalphysik), Universität des Saarlandes, Campus E2.6, 66123 Saarbrücken, Germany. ${ }^{3}$ Element Six Ltd., Global Innovation Centre, Fermi Avenue, Harwell Oxford, Didcot OX11 OQR, UK. Correspondence and requests for materials should be addressed to B.P. (email: bjpp2@cam.ac.uk) or to M.A. (email: ma424@cam.ac.uk).
} 
S pin-free materials, such as diamond and silicon, are ideal hosts to investigate and control the dynamics of spin-carrying defects. Among such systems, the nitrogenvacancy $(\mathrm{NV})$ centre in diamond has attracted great interest for electromagnetic field sensing ${ }^{1-4}$, bio-labelling ${ }^{5,6}$ and quantum information processing ${ }^{7-11}$. However, the $\mathrm{NV}$ centre only emits around $4 \%$ of its fluorescence into the zero-phonon line, limiting its use for quantum information processing in the absence of elaborate photonic structures such as photonic cavities ${ }^{12}$. In contrast, the negatively charged silicon-vacancy centre in diamond $\left(\mathrm{SiV}^{-}\right)$emits around $80 \%$ of its photons into the zero-phonon line ${ }^{13}$, with optical properties characterized by spectral stability and narrow inhomogeneous distribution in bulk diamond ${ }^{14}$. This makes the $\mathrm{SiV}^{-}$an ideal building block for a distributed quantum network ${ }^{15,16}$. Furthermore, the $\mathrm{SiV}^{-}$offers two possible realizations of a quantum bit: one consists in using the two readily available orbital branches of the ground state, split by $50 \mathrm{GHz}$ (ref. 17). The other takes advantage of the $\mathrm{SiV}^{-}$ground-state spin $S=1 / 2$, optical signatures of which have previously been reported ${ }^{18}$ and which promises longer coherence times, as estimated through coherent population trapping ${ }^{19,20}$. Using the spin as a quantum bit allows tuning of the resonance frequency with a magnetic field in the few $\mathrm{GHz}$ range, routinely used for NV centres. Achieving coherent control of this spin is a fundamental step for the implementation of spin-based quantum computing algorithms. Using microwave control provides more flexibility in the orientation of the magnetic field than all-optical control, which requires lambda-type transitions to be allowed. Furthermore, the capacity to address the spin state of the $\mathrm{SiV}^{-}$with microwave pulses is crucial to use the $\mathrm{SiV}^{-}$as an interface between optical and microwave photons for hybrid quantum computing ${ }^{21}$. Similar efforts are also undertaken with other colour centres in diamond ${ }^{22}$.

Here we report the realization of coherent control of the silicon-vacancy centre electronic spin. We perform optically detected magnetic resonance (ODMR) by tuning the frequency of a microwave field into resonance with the Zeeman splitting between two electronic spin states of a single $\mathrm{SiV}^{-}$centre. This allows us to perform spectroscopy of the magnetic dipolar transitions between ground-state levels of opposite electronic spin. By fixing the frequency of the microwave field in resonance with one of those transitions and varying the duration of the pulse, we coherently drive the $\mathrm{SiV}^{-}$electronic spin. We then perform Ramsey interferometry and measure a spin dephasing time of $115 \pm 9 \mathrm{~ns}$ at $3.6 \mathrm{~K}$. The temperature dependence of the spin coherence times reveals that spin dephasing and population decay are dominated by single-phonon excitation to the upper orbital branch of the ground state.

\section{Results}

Spin initialization and read-out. The $\mathrm{SiV}^{-}$centre is composed of a silicon atom replacing two neighbouring carbon atoms in the diamond lattice (Supplementary Note 1). Its energy levels are characterized by orbitally split ground and excited states (Fig. 1a). We study single $\mathrm{SiV}^{-}$centres in bulk diamond, created by implantation of isotopically purified ${ }^{29} \mathrm{Si}$ followed by annealing (see the 'Methods' section). An external magnetic field lifts the degeneracy of the electronic spin $S=1 / 2$, resulting in the energy level diagram shown in Fig. 1a. In our experiment, this magnetic field is applied at an angle of $109.5^{\circ} \pm 1^{\circ}$ with respect to the $\mathrm{SiV}^{-}$ symmetry axis, which dictates that all transitions between ground and excited states are optically allowed ${ }^{19}$. We use an optical pulse from a diode laser tuned to resonance with transition D1 (between ground-state level 1 and excited-state level D, red double arrow in Fig. 1a) to pump the $\mathrm{SiV}^{-}$optically into the spin-down ground state. Fluorescence from the $\mathrm{SiV}^{-}$is collected on the remaining transitions (solid grey arrows in Fig. 1a) following thermalization among excited states (dashed grey arrows in Fig. 1a). The decrease of fluorescence during the laser pulse (Fig. 1b) allows to extract an initialization fidelity of about $85 \%$ through a master equation model (Supplementary Note 5). We perform the spin read-out analogously by measuring the recovery of the leading edge of the fluorescence generated by a second optical pulse resonant with the same transition. The spinstate measurement corresponds to the peak ratio between the integrated areas under the leading edge of the read-out pulse and that of the initialization pulse. By varying the time delay between the two pulses, we extract from the exponential recovery a spin relaxation time $T_{1, \text { spin }}=350 \pm 11 \mathrm{~ns}$ (Fig. 1c) at $3.5 \mathrm{~K}$, setting an upper time bound for subsequent driving of the $\mathrm{SiV}^{-}$spin in this configuration.

Optically detected magnetic resonance. To address the electronic spin of the $\mathrm{SiV}^{-}$, we apply a microwave pulse between the optical initialization and read-out pulses (Fig. 2a). Owing to the nuclear spin $1 / 2$ of the ${ }^{29} \mathrm{Si}$ isotope, each electronic spin state displays a hyperfine splitting, as depicted in Fig. 2b. The microwave field drives the electronic spin, while preserving the nuclear spin state, which results in two distinct microwave resonance frequencies (orange and green circular arrows in Fig. 2b). When on resonance with one of the two transitions, the microwave transfers population from the initialized spin state to the depleted one, resulting in a recovery of fluorescence during the read-out pulse and thus a peak in the ODMR spectrum with a contrast around 33\% (Fig. 2c and Supplementary Note 3). We measure the frequencies of the two resonances at different values of the applied magnetic field, as shown in Fig. $2 \mathrm{~d}$. We fit the observed frequency shift using an effective Hamiltonian described in ref. 23, which includes the Jahn-Teller effect, spin-orbit coupling and electronic Zeeman effect, and accounts for the nuclear spin via the nuclear Zeeman effect and the hyperfine interaction (Supplementary Note 4). From the fit, we extract a value of $A_{\|}=70 \pm 2 \mathrm{MHz}$ for the hyperfine constant along the $\mathrm{SiV}^{-}$axis, in agreement with previously reported experimental $^{20,24}$ and theoretical ${ }^{25,26}$ values (Supplementary Note 4).

Coherent control of the spin. Having determined the frequencies of the electron spin-flipping transitions, we fix the microwave frequency in resonance with one of them and vary the duration of the microwave pulse, as illustrated in Fig. 3a. Figure $3 \mathrm{~b}$ shows the evolution of the read-out to initialization peak ratio with respect to the microwave pulse duration when the microwave pulse is resonant with the transition between states $\left|\downarrow, \mathrm{n}_{\uparrow}\right\rangle$ and $\left.\uparrow \uparrow, \mathrm{n}_{\uparrow}\right\rangle$ (green dots) and when it is resonant with the transition between states $\left|\downarrow, n_{\downarrow}\right\rangle$ and $\left|\uparrow, n_{\downarrow}\right\rangle$ (orange dots). The signal displays Rabi oscillations, sign of the coherent driving of the electronic spin of the $\mathrm{SiV}^{-}$(the nuclear spin orientation remaining unchanged). The upward drift of the oscillations arises from spinpreserving population transfers between the two orbital branches of the ground state. This is confirmed by an eight-level master equation model (Supplementary Note 5), used to reproduce the experimental curve (green and orange curves in Fig. 3b). This phenomenon highlights the importance of inter-branch dynamics at temperatures around a few kelvins. The detuned driving of the electron spin associated with the other nuclear spin orientation and of the transitions in the upper branch of the ground state (containing approximately one-third of the population at $4 \mathrm{~K}$ ) is responsible for a decreased contrast of the oscillations and their slightly irregular shape (Supplementary Figs 7 and 8). The linear 
a

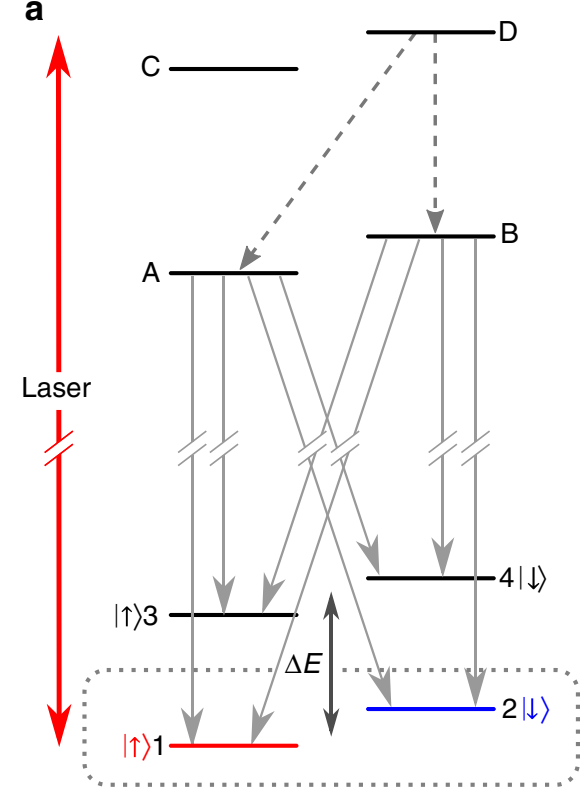

b

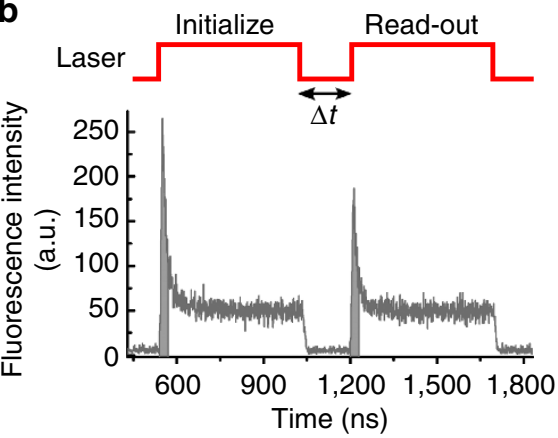

C

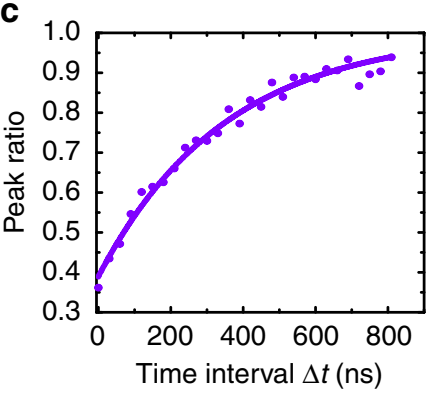

Figure 1 | Spin initialization and read-out. (a) Energy level scheme for a SiV - under magnetic field. Excited-state levels are labelled from A to D. States labelled 3 and 4 correspond to the upper orbital branch of the ground state. The dotted grey box highlights the Zeeman-split lower orbital branch, on which we focus in this work and where state 1 (in red) corresponds to a spin-up state and state 2 (in blue), to a spin-down state. The ground state upper orbital branch lies approximately at $\Delta E=50 \mathrm{GHz}$ above the lower one. A tunable laser at a wavelength of $\sim 737 \mathrm{~nm}$ (red double arrow) drives transition D1 resonantly. Grey dashed arrows show fast decay paths from excited state D to excited states A and B. Solid grey arrows identify the measured fluorescent transitions. (b) A first 500 ns-long optical pulse resonant with transition D1 causes optical pumping into the spin-down state, as evidenced by the exponential decay of the fluorescence. The height of the leading edge of the fluorescence due to the second 500 ns-long pulse indicates the recovery of the spin-up population, and thus acts as a read-out. The dark grey areas under the leading edges of the initialization and read-out pulses correspond to a duration of 30 ns during which the signal is integrated to calculate the peak ratio. (c) Recovery of the ratio between the leading edge of the read-out pulse with respect to the initialization pulse as a function of the time interval between the pulses at $3.5 \mathrm{~K}$ (purple dots). The purple curve is an exponential fit with $1 /$ e value $T_{1, \text { spin }}=350 \pm 11 \mathrm{~ns}$.
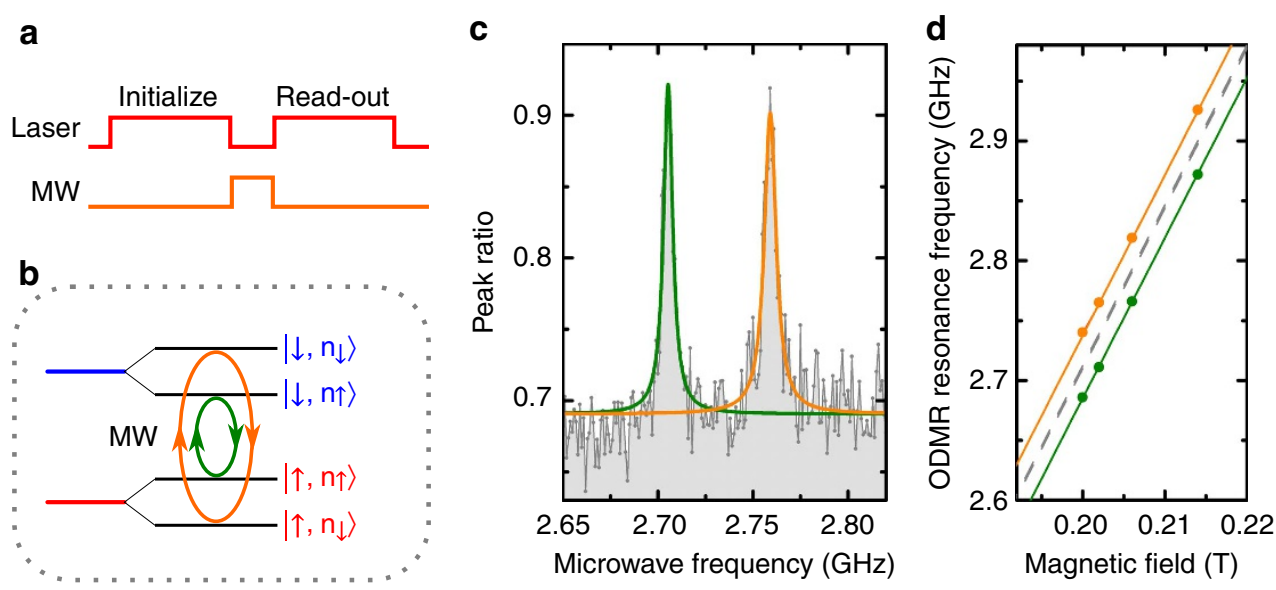

Figure 2 | Optically detected magnetic resonance. (a) ODMR pulse scheme. A $140 \mathrm{~ns}$ microwave pulse is applied during the $160 \mathrm{~ns}$ interval between optical initialization and read-out pulses at a measured temperature of $4.3 \mathrm{~K}$. (b) Close-up on the Zeeman-split lower branch of the ground state. The hyperfine interaction with the ${ }^{29} \mathrm{Si}$ nuclear spin $1 / 2$ causes a splitting of each electronic spin state. An applied microwave can flip the electronic spin, leaving the nuclear spin unchanged, resulting in two possible resonance frequencies (orange and green circular arrows). (c) ODMR spectrum: the peak ratio is plotted as a function of the microwave frequency. The two microwave resonances appear as two peaks, each fitted with a Lorentzian function (colours correspond to those of $\mathbf{b}$ ) and separated by $53.7 \pm 0.3 \mathrm{MHz}$ (error from Lorentzian fit). The ODMR contrast calculated as the ratio between the peak height and the baseline is about 33\%. (d) ODMR resonance frequencies as a function of external magnetic field (orange and green dots). Error bars are smaller than dots and correspond to the s.d. of $\sim 0.2 \mathrm{MHz}$ on the central frequency of the ODMR peak Lorentzian fits. The solid curves are fits based on the model developed in ref. 23 expanded to include the nuclear spin. Orange and green curves correspond to the transitions in $\mathbf{b}$, the two overlapping dashed grey curves correspond to the transitions where both electronic and nuclear spins are flipped. 
a

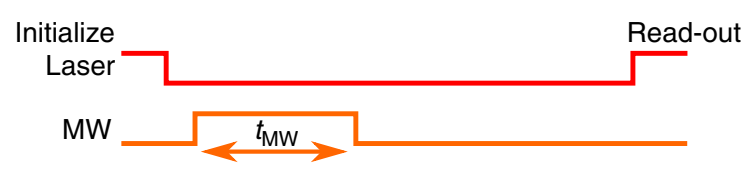

b
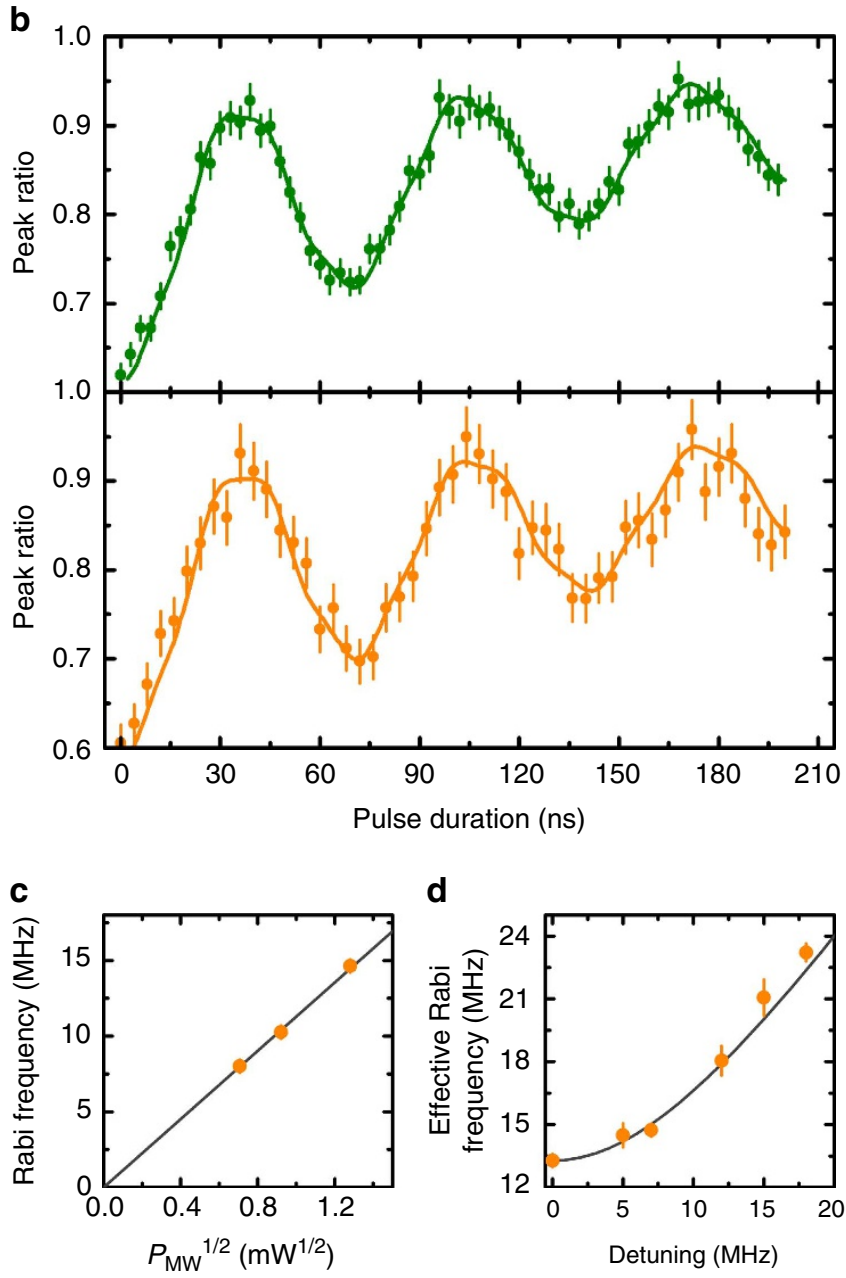

Figure 3 | Rabi oscillations. (a) Microwave and optical pulses scheme: the frequency of the microwave is fixed at one of the microwave resonances and its duration $t_{\mathrm{MW}}$ is varied. The time interval between initialization and read-out pulses is kept constant at $210 \mathrm{~ns}$, not to include the effect of the spin population decay into the measured signal. (b) Rabi oscillations between states $\left|\downarrow, n_{\uparrow}\right\rangle$ and $\left|\uparrow, n_{\uparrow}\right\rangle$ (green dots) and between states $\left|\downarrow, n_{\downarrow}\right\rangle$ and $\left|\uparrow, n_{\downarrow}\right\rangle$ (orange dots) (corresponding to the green and orange circular arrows in Fig. $2 b$, respectively): the measured peak ratio between the leading edges of the read-out and initialization pulses oscillates as a function of the microwave pulse duration. The error bars correspond to the s.d. of the peak ratio (the difference in error bars between the two graphs is due to different integration times (see Methods)). The green (respectively orange) solid curve is a fit based on an eight-level master equation model (Supplementary Note 5). (c) Evolution of the Rabi frequency as a function of the square root of the input microwave power $P_{M W}$, measured between states $\left|\downarrow, n_{\downarrow}\right\rangle$. and $\left|\uparrow, n_{\downarrow}\right\rangle$. The error bars are smaller than dots. The grey line is a linear fit. (d) Effective Rabi frequency as a function of microwave frequency detuning, measured on transition $\left|\downarrow, n_{\downarrow}\right\rangle$ and $\left|\uparrow, n_{\downarrow}\right\rangle$ (orange dots). The error bars are the s.d. of the Rabi frequencies extracted from fits. The grey line is a fit of the form $\sqrt{\Omega^{2}+\delta^{2}}$, where $\Omega$ is the bare Rabi frequency and $\delta$ is the detuning.

dependence of the Rabi frequency with the square root of the microwave power (Fig. 3c), as well as its variation with respect to detuning (Fig. 3d) confirms the coherent nature of those a
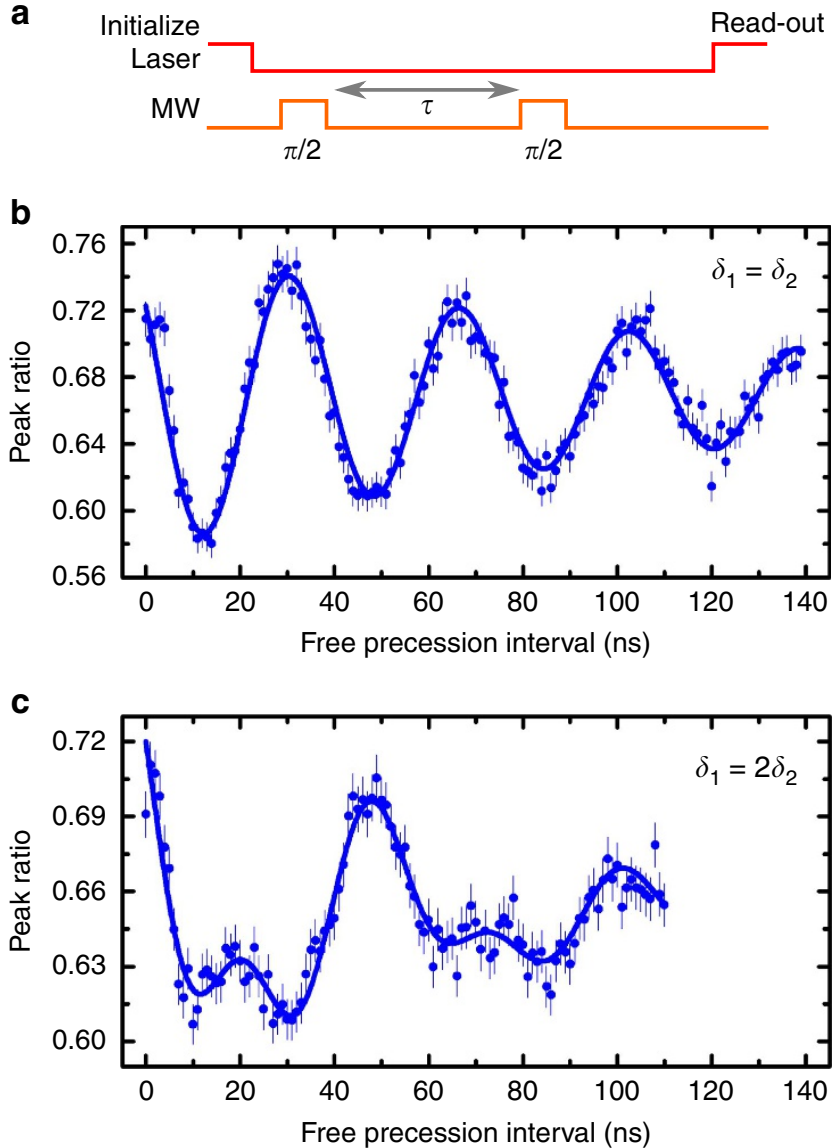

Figure 4 | Ramsey interferometry. (a) Microwave and optical pulses protocol: two microwave $\pi / 2$ pulses of $8 \mathrm{~ns}$ each are separated by a variable delay $\tau$. The time interval between the initialization and read-out pulses is held constant at $160 \mathrm{~ns}$, not to include the effect of the spin population decay into the signal. (b) Variation of the peak ratio as a function of the delay between the microwave pulses (blue dots) at $3.6 \mathrm{~K}$ (error bars correspond to the s.d. of the peak ratio). The microwave frequency is fixed at the average of the two resonance frequencies (the detuning $\delta_{1}$ from the lower frequency transition equals the detuning $\delta_{2}$ from the other). The blue solid curve is a fit of the form $\cos (2 \pi \cdot \tau \cdot \delta) \exp \left(-\tau / T_{2}{ }^{*}\right)$, where $\delta$ is the common detuning between the microwave frequency and the two resonances. We extract $T_{2}{ }^{\star}=115 \pm 9 \mathrm{~ns}$ (the error corresponds to the s.d. on the fit parameter). (c) The duration of the microwave pulses is kept unchanged compared to $\mathbf{b}$, but the frequency is tuned such that the detuning from one resonance is twice that with the other $\left(\delta_{1}=36 \mathrm{MHz}\right.$ and $\delta_{2}=18 \mathrm{MHz}$ ). The solid curve is a fit where the cosine function in $\mathbf{b}$ is replaced by the sum of two cosine functions, with frequencies corresponding to the respective detunings.

oscillations. The observed bare Rabi frequency of about $15 \mathrm{MHz}$ is comparable to what is obtained with $\mathrm{NV}^{-}$centres in similar conditions.

Ramsey interferometry. To measure the free induction decay time of the spin directly, we perform microwave Ramsey interferometry. We use a pulse sequence consisting of two $\pi / 2$ microwave pulses separated by a variable delay (Fig. 4a). We fix the frequency of the microwave pulses such that in one case (Fig. 4b), the microwave frequency is in the middle of the two resonances, corresponding to a detuning of about $27 \mathrm{MHz}$ from each of them, and in the other case (Fig. 4c), the detuning from one resonance equals twice that from the other (36 and 
$18 \mathrm{MHz}$, respectively). The measured peak ratio displays oscillations as a function of the free precession interval separating the two pulses. Since the frequency of Ramsey fringes corresponds to the value of the detuning of the microwave frequency, in Fig. $4 \mathrm{~b}$, the oscillations due to both resonances add constructively, while in Fig. 4c, they display a beating signal between two frequencies with one being twice the other. The decay of the Ramsey fringes gives a direct measurement of the dephasing time of the electronic spin $T_{2}{ }^{*}=115 \pm 9 \mathrm{~ns}$ at $3.6 \mathrm{~K}$. This value is commensurate with the characteristic time of population transfer between the two orbital branches of the ground state, taken as twice the orbital decay time $2 T_{1, \text { orbital }}=133 \pm 4 \mathrm{~ns}$ at $3.6 \mathrm{~K}$ in our measurements. Shorter dephasing times have previously been obtained at slightly higher temperatures through coherent population trapping ${ }^{19,20}$, which provides a lower bound due to additional sources of dephasing, including noise from the driving fields. A straininduced increase of orbital splitting to an energy at which phonon population is larger ${ }^{27}$ is also a cause for shorter dephasing times (see below).

Single-phonon-mediated spin dephasing and decay. The strongest candidate for the cause of limited $T_{2}{ }^{*}$ is expected to be phonon excitations from the lower to the upper orbital branches in the ground-state manifold. To verify this directly, we measure the spin dephasing rate $1 / T_{2}{ }^{*}$ as a function of temperature, as shown in Fig. 5a as blue dots. At the temperatures measured in the experiment, the orbital transition rate from the lower to the upper branch of the ground state is expected to be lower than that from the upper to the lower branch by a Boltzmann factor (Supplementary Note 5). Since in our set-up configuration, we underestimate the absolute temperature at the $\mathrm{SiV}$, we take $1 /\left(2 T_{1, \text { orbital }}\right)$ as a reference for the orbital decay rate and display it as grey dots in Fig. 5a. The orbital decay rate is obtained in the absence of magnetic field similarly to the spin population decay presented in Fig. 1b,c, with initialization into the lower orbital branch of the ground state and measurement of the population recovery into the upper branch (Supplementary Note 6). The orbital decay rate increases linearly with temperature, a signature attributed to single-phonon excitation process between the two orbital branches of the ground state $e^{20,28}$. The spin dephasing rate follows closely the orbital decay rate dependence on temperature, manifesting that spin dephasing is dominated by single-phonon- mediated transitions between the orbital branches. We also measure the temperature dependence of the spin decay rate $1 /\left(2 T_{1, \text { spin }}\right)$, as shown in Fig. 5b. Its linear dependence with temperature indicates that it is also dominated by a singlephonon process. The suggested mechanism responsible for spin decay relies on excitation to the upper orbital branch of the ground state through the absorption of a single phonon followed by decay back to the lower branch. Owing to a slight mismatch of spin quantization axes between the two orbitals (Supplementary Note 4), such transitions can result in a spin flip. This mechanism also explains why $T_{1 \text {,spin }}$ can reach several milliseconds ${ }^{20}$ when the magnetic field is aligned with the SiV symmetry axis, which otherwise constitutes a competing quantization axis for the spin via the spin-orbit interaction ${ }^{19,23}$. The phonons responsible for those decoherence mechanisms have a frequency around $50 \mathrm{GHz}$ corresponding to the orbital splitting of the $\mathrm{SiV}^{-}$and still have a significant population around $4 \mathrm{~K}$.

\section{Discussion}

In conclusion, we have achieved coherent control of a single $\mathrm{SiV}^{-}$spin with a microwave field, following the identification of spin-flipping transitions through ODMR. We have subsequently implemented Ramsey interferometry to get direct access to the spin dephasing time. The temperature dependence of the dephasing and decay rates reveal that both processes are governed by excitation from the lower to the upper orbital branch of the ground state through single-phonon absorption. This indicates that the coherence times of the $\mathrm{SiV}^{-}$spin will improve significantly by cooling the system to lower temperatures. An alternative approach consists in splitting the orbital branches further apart by applying strain to the $\mathrm{SiV}^{-}$, thus increasing the energy required for phonons to cause decoherence. Those approaches would not necessarily improve significantly the coherence time of the orbital-based qubit, which is limited by phonon absorption and emission processes, simultaneously. The latter could be addressed by decreasing the phonon density of states around the frequency of the orbital splitting, which most likely requires nanostructures with dimensions smaller than about $120 \mathrm{~nm}$, corresponding to half the phonon wavelength. Microwave control of the spin offers a strong advantage compared to all-optical control in that it gives much more flexibility for the orientation of the external magnetic field with
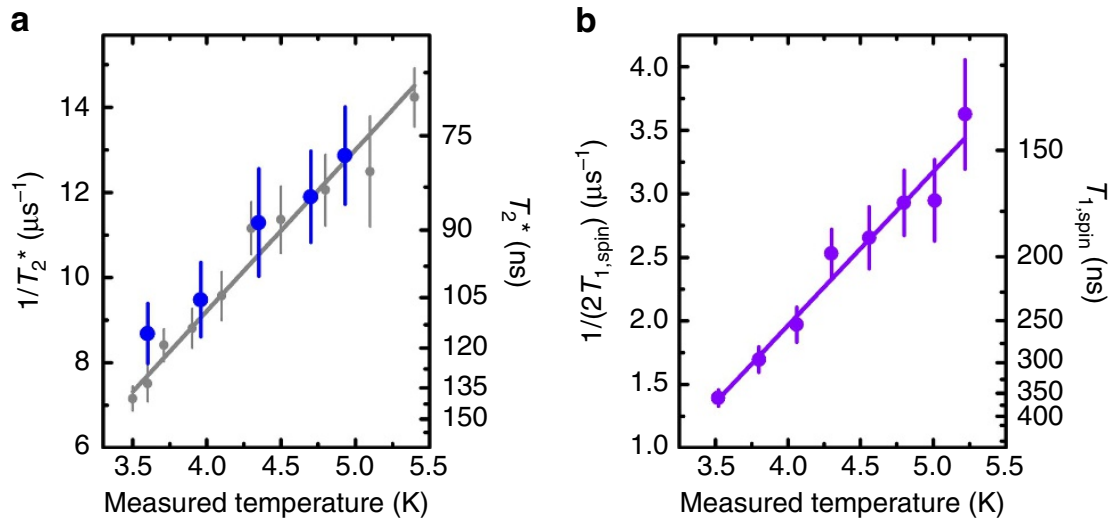

Figure 5 | Phonon-induced spin dephasing and decay. (a) Evolution of the spin dephasing rate $1 / T_{2}{ }^{\star}$ (blue dots) and orbital population decay rate taken as $1 /\left(2 T_{1, \text { orbital }}\right)$ (grey dots) as a function of temperature. The solid grey curve is a linear fit of the variation of $1 /\left(2 T_{1, \text { orbital }}\right)$. (b) Temperature dependence of the spin decay rate $1 /\left(2 T_{1, \text { spin }}\right)$. The solid purple curve is a linear fit of the experimental values. In a,b, the error bars correspond to the s.e.s. on the fit parameter $T_{2}{ }^{*}, T_{1, \text { orbital }}$ and $T_{1, \text { spin, }}$ respectively. In both figures, the $y$ axis on the right indicates the coherence times corresponding to the rates of the left axis. The temperature is measured below the sample mount, and, due to heating from the microwave, is expected to be lower than the temperature at the SiV ${ }^{-}$. 
respect to the $\mathrm{SiV}^{-}$symmetry axis. In particular, control through microwave pulses allows to bring the magnetic field axis close to alignment with the $\mathrm{SiV}^{-}$axis, thus giving access to cycling optical transitions ${ }^{19}$ for single-shot read-out of the spin state ${ }^{29}$. The combination of the optical qualities of the $\mathrm{SiV}^{-}$with the microwave addressing of some of its states also make the $\mathrm{SiV}^{-}$a promising system to be used as a quantum transducer between optical and microwave photons ${ }^{21}$. Finally, the nuclear spin associated with ${ }^{29} \mathrm{Si}$ can offer the opportunity of a quantum register, if it can be controlled coherently using radio-frequency pulses and hyperfine coupling to the electron spin.

\section{Methods}

Sample preparation. The sample used for this experiment is a high-pressure high-temperature type IIa bulk diamond (Element Six) with surface oriented orthogonally to the [111] crystallographic axis. $\mathrm{SiV}^{-}$centres were subsequently created by ion implantation of isotopically purified ${ }^{29} \mathrm{Si}^{+}$ions at an implantation energy of $900 \mathrm{keV}$. Simulations using the stopping range of ions in matter algorithm ${ }^{1}$ showed that the $\mathrm{Si}^{+}$stop at $500 \pm 50 \mathrm{~nm}$ below the diamond surface. Implantation doses were tuned from one implantation site to the other from $10^{9}$ to $10^{12}$ ions per $\mathrm{cm}^{2}$. Following the implantation, the sample was annealed at $1,000^{\circ} \mathrm{C}$ in vacuum for $3 \mathrm{~h}$, followed by an oxidation step in air for $1 \mathrm{~h}$ at $460^{\circ} \mathrm{C}$. Single $\mathrm{SiV}^{-}$centres have been found in the $10^{10}$ ions per $\mathrm{cm}^{2}$ area.

Experimental set-up. The diamond sample is mounted in a closed-cycle liquid helium cryostat (Attodry 1000) reading $3.5 \mathrm{~K}$ at the sample and the temperature can be tuned via a resistive heater positioned under the sample mount or using heating caused by the microwave pulses. A superconducting coil around the sample space allows to apply a vertical magnetic field from 0 to $9 \mathrm{~T}$. The optical part of the set-up consists of a home-built confocal microscope mounted on top of the cryostat and a microscope objective with numerical aperture $=0.82$ inside the sample chamber. The sample is moved with respect to the objective utilizing piezoelectric stages (Attocube) on top of which the sample is mounted. $\mathrm{SiV}^{-}$excitation is performed using a frequency tunable diode laser (Toptica DL pro design) whose frequency is maintained in resonance with an optical transition of the $\mathrm{SiV}^{-}$ through continuous feedback from a wavemeter (High Finesse WSU). Optical pulses are generated with an acousto-optic modulator (AAOptoelectronic MT350-A0.12-800) controlled by a delay generator (Stanford Research Instruments DG645). The fluorescence from the $\mathrm{SiV}^{-}$is collected through the same objective. The laser light and $\mathrm{SiV}^{-}$fluorescence from the excited transition are filtered out using a home-built monochromator, and the fluorescence from the remaining transitions is sent to an avalanche photodiode (PicoQuant Tau-Spad) The signal from the avalanche photodiode is sent to a time-to-digital converter (qutools quTAU) triggered by the delay generator. The microwave is generated by a frequency generator (Stanford Research Instruments SG384), pulses are generated with a switch connected to the delay generator used for optical pulses. Microwave pulses are then amplified by a microwave amplifier (Mini-Circuits ZHL-16W-43-S + ). They travel through semi-rigid cables installed inside the cryostat. A single copper wire $(20 \mu \mathrm{m}$ diameter $)$ positioned on top of the sample allows the microwave to be radiated to $<20 \mu \mathrm{m}$ away from the studied $\mathrm{SiV}^{-}$.

Experimental details. The results presented in Fig. $1 \mathrm{~b}$ have been acquired with a repetition rate of $500 \mathrm{kHz}$ and an integration time of $300 \mathrm{~s}$. Those in Fig. $2 \mathrm{c}$ were obtained with a repetition rate of $500 \mathrm{kHz}$ and an integration time of $200 \mathrm{~s}$ per data point. The results in Fig. $3 \mathrm{~b}$ were recorded with a repetition rate of $20 \mathrm{kHz}$ to minimize heating from the microwave and an integration time of 7,200 s per data point for the green curve and 3,000 s per data point for the orange curve. Finally, the results in Fig. $4 \mathrm{~b}, \mathrm{c}$ were measured with a repetition rate of $50 \mathrm{kHz}$ (to minimize heating) with $2,000 \mathrm{~s}$ integration and $200 \mathrm{kHz}$ with $400 \mathrm{~s}$ integration, respectively.

Data availability. The data that support the findings of this study are available from the corresponding author on reasonable request.

\section{References}

1. Degen, C. L. Scanning magnetic field microscope with a diamond single-spin sensor. Appl. Phys. Lett. 92, 243111 (2008).

2. Taylor, J. et al. High-sensitivity diamond magnetometer with nanoscale resolution. Nat. Phys. 4, 810-816 (2008).

3. Dolde, F. et al. Electric field sensing using single diamond spins. Nat. Phys. 7, 459-463 (2011).

4. Grinolds, M. S. et al. Nanoscale magnetic imaging of a single electron spin under ambient conditions. Nat. Phys. 9, 215-219 (2013).

5. Mohan, N. et al. In vivo imaging and toxicity assessments of fluorescent nanodiamonds in Caenorhabditis elegans. Nano Lett. 10, 3692-3699 (2010).
6. Hegyi, A. \& Yablonovitch, E. Molecular imaging by optically detected electron spin resonance of nitrogen-vacancies in nanodiamonds. Nano Lett. 13, 1173-1178 (2013).

7. Wrachtrup, J. \& Jelezko, F. Processing quantum information in diamond. J. Phys. Condens. Matter 18, S807-S824 (2006).

8. Neumann, P. et al. Quantum register based on coupled electron spins in a room temperature solid. Nat. Phys. 6, 249-253 (2010).

9. Bernien, H. et al. Heralded entanglement between solid-state qubits separated by three metres. Nature 497, 86-90 (2013).

10. Pfaff, W. et al. Unconditional quantum teleportation between distant solid-state quantum bits. Science 345, 532-535 (2014).

11. Hensen, B. J. et al. Loophole-free Bell inequality violation using electron spins separated by 1.3 kilometres. Nature 526, 682-686 (2015).

12. Riedrich-Möller, J. One- and two-dimensional photonic crystal microcavities in single crystal diamond. et al. Nano Lett. 14, 5281-5287 (2014).

13. Neu, E. et al. Single photon emission from silicon-vacancy colour centres in chemical vapour deposition nano-diamonds on iridium. New J. Phys. 13, 025012 (2011).

14. Sipahigil, A. et al. Indistinguishable photons from separated silicon-vacancy centers in diamond. Phys. Rev. Lett. 113, 113602 (2014).

15. Kimble, H. J. The quantum internet. Nature 453, 1023-1030 (2008).

16. Sipahigil, A. et al. An integrated diamond nanophotonics platform for quantum optical networks. Science 354, 847-850 (2016).

17. Becker, J. N. et al. Ultrafast all-optical coherent control of single silicon vacancy colour centres in diamond. Nat. Commun. 7, 13512 (2016).

18. Müller, T. et al. Optical signatures of silicon-vacancy spins in diamond. Nat. Commun. 5, 3328 (2014).

19. Pingault, B. et al. All-optical formation of coherent dark states of siliconvacancy spins in diamond. Phys. Rev. Lett. 113, 263601 (2014).

20. Rogers, L. J. et al. All-optical initialization, readout, and coherent preparation of single silicon-vacancy spins in diamond. Phys. Rev. Lett. 113, 263602 (2014)

21. Williamson, L. A. et al. Magneto-optic modulator with unit quantum efficiency. Phys. Rev. Lett. 113, 203601 (2014).

22. Siyushev, P. et al. Optical and microwave control of germanium-vacancy center spins in diamond. Preprint at https://arxiv.org/abs/1612.02947 (2016)

23. Hepp, C. et al. Electronic structure of the silicon vacancy color center in diamond. Phys. Rev. Lett. 112, 036405 (2014).

24. Edmonds, A. M. et al. Electron paramagnetic resonance studies of siliconrelated defects in diamond. Phys. Rev. B 77, 245205 (2008).

25. Goss, J. P. et al. Density functional simulations of silicon-containing point defects in diamond. Phys. Rev. B 76, 075204 (2007).

26. Gali, A. \& Maze, J. R. Ab initio study of the split silicon-vacancy defect in diamond: electronic structure and related properties. Phys. Rev. B 88, 235205 (2013).

27. Arend, C. et al. Photoluminescence excitation and spectral hole burning spectroscopy of silicon vacancy centers in diamond. Phys. Rev. B 94, 045203 (2016).

28. Jahnke, K. D. et al. Electron-phonon processes of the silicon-vacancy centre in diamond. New J. Phys. 17, 043011 (2015).

29. Robledo, L. et al. High-fidelity projective read-out of a solid-state spin quantum register. Nature 477, 574-578 (2011)

\section{Acknowledgements}

We acknowledge financial support by the University of Cambridge, the ERC Grant PHOENICS, FP7 Marie Curie Initial Training Networks $S^{3}$ NANO and SPIN NANO, and the NQIT programme. This research has been partially funded by the European Community's Seventh Framework Programme (FP7/2007-2013) under Grant agreement no. 611143 (DIADEMS). B.P. thanks Wolfson College (Cambridge) for support through a Research Fellowship. Ion implantation was performed at and supported by RUBION, the central unit of the Ruhr-Universität Bochum. We thank D. Rogalla for the implantation, and C. Pauly for SIL fabrication. We thank M. Gündoğan, C. Stavrakas, A. Gali, J. Beitner, D. Kara, H.S. Knowles and Y. Kubo for helpful discussions.

\section{Author contributions}

M.A. and C.B. supervised the project; M.M., J.N.B. and C.B. prepared the sample; B.P., D.-D.J., C.H., L.K. and J.N.B. set up the experimental apparatus; B.P. conducted the experiments and analysis of the results, designed the theoretical models, and wrote the manuscript. All authors participated in the writing of the manuscript.

\section{Additional information}

Supplementary Information accompanies this paper at http://www.nature.com/ naturecommunications

Competing interests: The authors declare no competing financial interests. 
Reprints and permission information is available online at http://npg.nature.com/ reprintsandpermissions/

How to cite this article: Pingault, B et al. Coherent control of the silicon-vacancy spin in diamond. Nat. Commun. 8, 15579 doi: 10.1038/ncomms15579 (2017).

Publisher's note: Springer Nature remains neutral with regard to jurisdictional claims in published maps and institutional affiliations. (c) (i) This work is licensed under a Creative Commons Attribution 4.0 International License. The images or other third party material in this article are included in the article's Creative Commons license, unless indicated otherwise in the credit line; if the material is not included under the Creative Commons license, users will need to obtain permission from the license holder to reproduce the material. To view a copy of this license, visit http://creativecommons.org/licenses/by/4.0/

(C) The Author(s) 2017 